\title{
Characterization and optimization of liquid crystal displays for data storage applications
}

\author{
Andrés Márquez ${ }^{1}$, Sergi Gallego ${ }^{1}$, David Méndez ${ }^{1}$, Mariela L. Álvarez ${ }^{1}$, Elena Fernández², \\ Manuel Ortuño ${ }^{1}$, Augusto Beléndez ${ }^{1}$, Inmaculada Pascual ${ }^{2}$ \\ ${ }^{1}$ Depto. de Física, Ingeniería de Sistemas y Teoría de la Señal, Universidad de Alicante, Spain \\ ${ }^{2}$ Departamento Interuniversitario de Óptica, Universidad de Alicante, Spain \\ Tel.: +34-96-5903400 (ext. 2543); Fax: +34-96-5909750; E-mail: Andres.Marquez@ua.es
}

\begin{abstract}
We present the characterization and the optimization of the phase and amplitude modulation of a liquid crystal display (LCD) applied to holographic data storage. We discuss the main LCD modulation regimes demanded in the literature in the application to holographic memories: binary amplitude, binary phase and hybrid ternary modulation (HTM). We show how to obtain optimally these modulation regimes with a LCD. In our strategy it is essential the accuracy demonstrated by the model we use to describe the modulation properties of the LCD. This accuracy allows for a reliable computer search of the configurations enabling for an optimum LCD complex amplitude modulation. Results are given for the calibration of a commercial LCD. Optimum configurations close to the ideal for each of the modulation regimes are also obtained and the values of the various parameters characterizing the quality of these configurations are provided. We have also analyzed the performance of these configurations to perform the Fourier transform (FT) of a binary data page. We see that the optimum configurations obtained for the binary phase and the HTM regimes produce a homogeneous distribution of the energy in the Fourier plane with no DC peak.
\end{abstract}

Keywords: Liquid crystal displays, spatial light modulation, holographic data storage, phase and amplitude modulation, hybrid ternary modulation, binary modulation.

\section{INTRODUCTION}

Liquid crystal displays (LCDs) ${ }^{1}$ have been widely used as spatial light modulators (SLMs) in many applications in optics. LCDs allow to modify in real time the amplitude, phase and/or the state of polarization of a light beam. This can be used to design programmable diffractive optical elements (DOEs), such as lenses, apodizing filters, data pages, or simply binary gratings, useful in diffractive optics, imaging systems, or in holographic data storage ${ }^{2-6}$. In particular in holographic data storage, LCDs enable the real time update of the data page to be recorded in the holographic recording material.

To obtain an accurate control of the amplitude and phase of the wavefront it is important a proper calibration of the modulation capabilities of the $\mathrm{LCD}^{7}$. In general, commercially available LCDs are based on the twisted-nematic configuration $^{1}$, where twisted means that the LC molecules twist from the entrance to the exit face about $90^{\circ}$ (twist angle), and nematic refers to a particular phase in which LC materials are found. In TN-LCDs the amplitude and the phase modulations are coupled ${ }^{8}$. Present LCDs have a larger resolution, forcing a decrease in the thickness of the device to provide a faster response for the addressing of the pixels. The decrease in the thickness shortens the dynamic modulation range of the device. In Ref. 9 and 10 it was demonstrated an optimization procedure leading to amplitudeonly and phase-only modulations for thin-LCDs. This optimization procedure is based on an approximate model describing the physical properties of the $\mathrm{LCD}^{11}$, and on the use of elliptically polarized light and short wavelengths.

Liquid Crystals and Applications in Optics, edited by Milada Glogarova, Peter Palffy-Muhoray, Martin Copic Proc. of SPIE Vol. 6587, 658715, (2007) · 0277-786X/07/\$18 - doi: 10.1117/12.722647 
In holographic data storage systems (HDSS) LCDs are normally used as binary amplitude transparencies to display the data page. Then the Fourier transform (FT) of the data page is holographically recorded on a photosensitive material ${ }^{6}$. In this case the higher the contrast between the two transmittance levels, the lower the bit-error rate (BER) will be. However, binary absorption data pages produce highly inhomogeneous Fourier transforms, with a very energetic zero frequency in comparison with the higher frequencies, which carry most of the information of the signal. This high zero frequency may saturate the dynamic range of the material, thus producing a decrease in the signal to noise ratio in the reconstruction and limiting the accessible dynamic range ${ }^{12}$. Various alternative schemes have been proposed, such as recording the defocused FT (i.e. recording the Fresnel transform). However, this limits the performance of a holographic memory in one of its most powerful applications, i.e. as associative memories, and lowers the storage density. Another alternative proposed is to use random or pseudo-random phase masks to uniformize the FT spectrum ${ }^{12,13}$. In this case the main drawback is the stringent requirements for optical alignment between the phase mask and the LCD with subpixel accuracy. Partly, the problems given by the lack of homogeneity of the FT can be solved by using some other modulation schemes, such as hybrid ternary modulation $(\mathrm{HTM})^{14,15}$ or binary phase modulation ${ }^{16,17}$.

In this work we characterize and optimize the phase and amplitude modulation of a LCD applied to HDSS. First, in Section 2 we present the model that we use to calibrate the modulation properties of the LCD. Results for the LCD used in this work are provided. In Section 3 we show how to obtain the optimum configurations for the various modulation regimes interesting for HDSS with a focus on the binary phase and the HTM regimes. In Section 4 we analyze the performance of these optimum configurations to obtain the FT of a binary data page. Eventually, the main conclusions of the work are given in Section 5.

\section{CHARACTERIZATION OF A LIQUID CRYSTAL DISPLAY}

To calculate the modulation curves for the LCD we need to know a series of parameters. They can be divided in independent and dependent on the applied voltage ${ }^{1,11}$. The voltage independent parameters are the total twist angle $(\alpha)$, the orientation of the director axis at the entrance face $\left(\Psi_{\mathrm{D}}\right)$ and the value of the maximum birefringence $\left(\beta_{\max }\right)$. The voltage dependent parameters are related with the profile of the optical anisotropy across the LC layer and its variation with the applied voltage. We use an approximate model to describe this profile. In the last years, this model ${ }^{11}$ has been proven to predict very accurately the amplitude and phase modulation provided by LCDs ${ }^{3-5,7,9-11}$. This model takes into account that the molecules in the vicinity of the faces of the LCD are highly anchored to the alignment layer in the windows and can not reorient with the applied voltage ${ }^{1}$. The total thickness of the LCD can be divided in two edge layers with a thickness $d_{1}$ and a central layer with a thickness $d_{2}$, as given in Fig. 1 . Thus, the anisotropic properties across the LCD are simplified in two voltage dependent parameters, the birefringence in the central layer $(\beta)$ and the birefringence in the edge layers $(\delta)$, which are defined as,

$$
\beta=\pi \Delta n d_{2} / \lambda_{0} ; \delta=\pi \Delta n_{\max } d_{1} / \lambda_{0}
$$

where $\lambda_{0}$ is the wavelength of the light beam, $\Delta n$ is the difference between the extraordinary and ordinary index of refraction, where $\Delta n_{\max }$ is the maximum value. The birefringences depend on the applied voltage through the layer thicknesses $d_{I}(V)$ and $d_{2}(V)$, and the index difference $\Delta n(V)$.

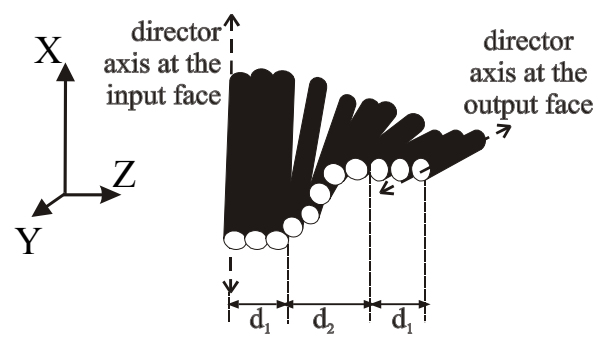

Figure 1. Proposed model for the TN-cell with the two edge layers of thickness $d_{l}$ and the central part of thickness $d_{2}$. 
Applying this model and using the Jones matriz formalism for polarized light we obtain the matrix $\mathrm{M}_{\mathrm{LCD}}$ describing the action of the LCD in transmission,

$$
\mathrm{M}_{L C D}=\exp (-j(\beta+2 \delta)) \mathrm{R}(-\alpha)\left(\begin{array}{cc}
X-j Y & Z \\
-Z & X+j Y
\end{array}\right)
$$

where $R(-\alpha)$ is the rotation matrix and,

$$
\begin{gathered}
X=\cos \gamma \cos (2 \delta)-\frac{\beta}{\gamma} \sin \gamma \sin (2 \delta) \\
Y=\cos \gamma \sin (2 \delta)+\frac{\beta}{\gamma} \sin \gamma \cos (2 \delta) \\
Z=\frac{\alpha}{\gamma} \sin \gamma \\
\gamma=\sqrt{\alpha^{2}+\beta^{2}}
\end{gathered}
$$

The expression in Eq. (2) considers the reference system with its X-axis parallel to the director axis at the entrance face of the LCD.

In this work we use a Sony LCD model LCX012BL, extracted from a video projector Sony VPL-V500. The Sony LCX012BL is a $3.3 \mathrm{~cm}$ diagonal active matrix thin film transistor (TFT) panel, with VGA resolution (640 x 480 pixels). The pixels are square with a pixel center to center separation of $41 \mu \mathrm{m}$ and a width of $34 \mu \mathrm{m}$. We use the electronics of the video projector to send the voltage to the pixels of the LCD.

The voltage independent parameters have been calculated by means of known techniques ${ }^{18-20}$. For these measurements we have used the unexpanded beams for two different wavelengths: $633 \mathrm{~nm}$ from a He-Ne laser and the 532 from a $\mathrm{Nd}: \mathrm{YVO}_{4}$ laser. In Table 1 we summarize the values obtained for the voltage independent parameters.

Table 1.Values of the parameters for the LCD used in the experiments.

\begin{tabular}{|c|c|c|c|}
\hline$\alpha\left(^{\circ}\right)$ & $\Psi_{\mathrm{D}}\left({ }^{\circ}\right)$ & $\beta_{\max }(\lambda=633 \mathrm{~nm})$ & $\beta_{\max }(\lambda=532 \mathrm{~nm})$ \\
\hline-92 & 47 & 135 & 171 \\
\hline
\end{tabular}

The voltage dependent parameters $\beta(V)$ and $\delta(V)$ are calculated using the technique proposed in Ref. 11 . We insert the TN-LCSLM between two linear polarizers with their transmission axes in specific orientations. The nonlinear curve fitting of intensity transmission measurements versus the applied voltage provide us with the values for $\beta(V)$ and $\delta(V)$. The experiments were conducted with the brightness and contrast controls of the video projector at 50 and 100 respectively to cover the widest possible range of applied voltages. The minimum and maximum values for these controls are 0 and 100 . We have measured the intensity at intervals of 10 gray scale levels. For the Sony VPL-V500 video projector electronics, the voltage decreases monotonically when the gray level increases.

In Fig. 2(a) and 2(b) we show the values obtained for the birefringences $\beta(V)$ and $\delta(V)$ at the two wavelengths as a function of the gray level. The $\beta$ birefringence (Fig. 2(a)) increases monotonically with the decrease in the applied voltage (increase in the gray level). The $\delta$ birefringence has its maximum value in the range of mid gray levels and is close to zero at low and at high gray levels. From the model in Ref. 11 it makes sense that the $\delta$ birefringence is close to zero at low values of applied voltage (high gray levels) since at this range the edge layers disappear. The result at low gray levels (high voltage) needs further discussion. It is a result partly unexpected from the interpretation proposed for the model in Ref. 11, since it predicts the increase of the $\delta$ birefringence with the applied voltage. However, if we interpret more carefully ${ }^{21}$ the origin of the expression for the $\delta$ birefringence (Eq. (1)) we see that $\delta$ is a global phase parameter with two contributions that can not be distinguished one from each other. One of the contributions is the 
average profile of the index difference $\Delta n$ in the vicinity of the faces (it is very restrictive to consider only the short length vicinity where the molecules are totally anchored, thus exhibiting $\Delta n_{\max }$ ). The other contribution is the thickness of these edge layers $d_{1}$. This thickness would have two different values depending if we look at the realistic profile across the LC layer for the twist or for the tilt angle $e^{1,22,23}$. In the twist profile the thickness increases with the applied voltage, and in the tilt profile it is not so clearly defined. In conclusion, a more rigorous interpretation of the model ${ }^{11}$ used in this work is compatible with the decrease of the $\delta$ birefringence at high voltage. This result has also been found in another liquid crystal device ${ }^{7}$. It will be demonstrated in Section 3 that the values for the birefringences in Fig. 2 are able to predict with a high accuracy the amplitude and phase modulation curves for the LCD, thus validating the model ${ }^{11}$ we use.
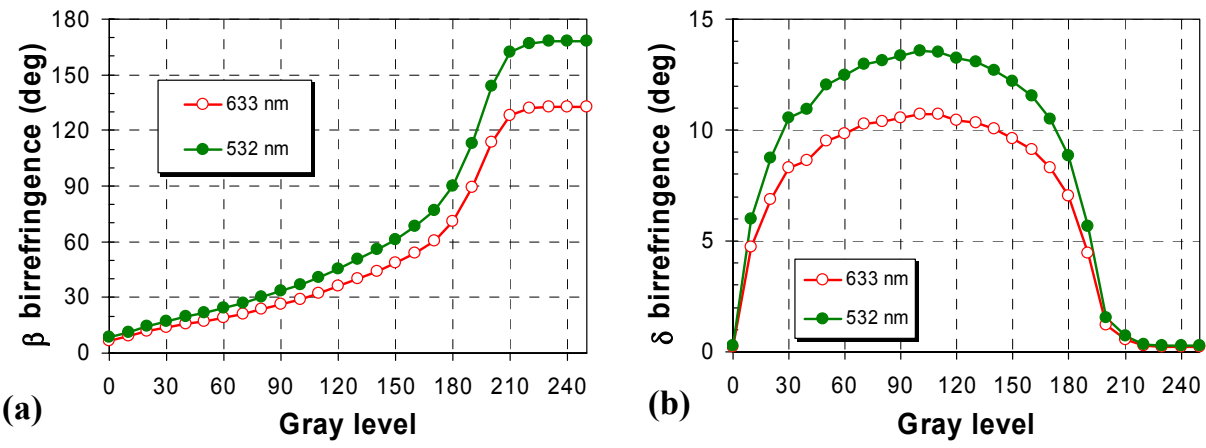

Figure 2. Values obtained for the voltage dependent parameters at the two wavelengths. (a) $\beta(V)$, (b) $\delta(V)$.

\section{OPTIMUM MODULATION CURVES FOR HOLOGRAPHIC DATA STORAGE}

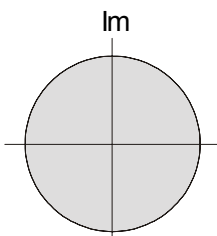

(a)

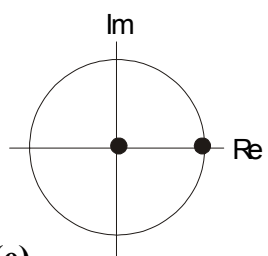

(e)

(f)

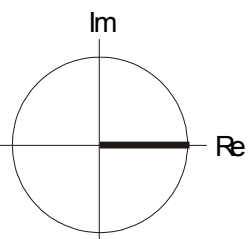

(b)

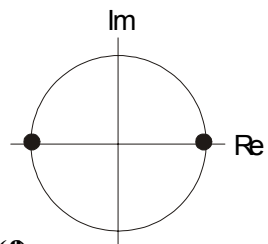

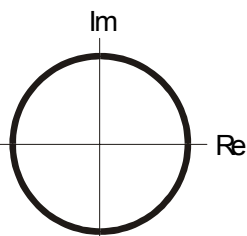

(c)

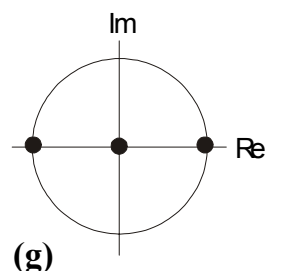

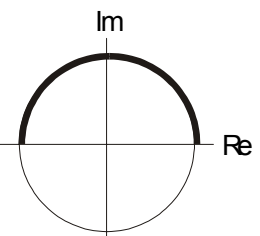

(d)

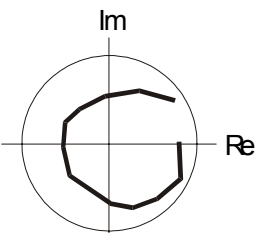

(h)

Figure 3. Typical modulation domains. (a) Ideal full range. (b) Amplitude-only. (c) Phase-only modulation with $2 \pi$ depth. (d)

Phase-only modulation with $\pi$ depth. (e) Binary amplitude. (f) Binary phase. (g) Ternary. (h) Coupled amplitude-phase.

In Fig. 3 we show a diagram of several modulation schemes useful in a number of different applications ${ }^{8}$. Availability of SLMs providing full complex modulation (Fig. 3(a)) would be the ideal situation since any other modulation is also included. LCDs provide coupled amplitude and phase modulation ${ }^{8}$ (Fig. 3(h)) which in general is not an interesting scheme (in this work we prove its applicability to HTM). With an appropriate knowledge of the variation the optical anisotropy in the LCD as a function of the applied voltage (or gray level) it is possible to obtain interesting modulation domains as presented from Fig. 3(b) to 3(g). In this sense the model presented in Section 2 provides accurate calculations of the modulation capability of the LCD. Using this model we can calculate, through an optimization procedure, the orientation of the polarizing devices in front and behind the LCD to obtain optimum amplitude-only (Fig. 3(b)) or phaseonly (Fig. 3(c)) modulations ${ }^{9,10}$. These are useful modulation domains in optical signal processing and in diffractive optics. In general with present LCDs, which are thin devices, we need to use elliptically polarized light to obtain 
amplitude-only and, especially, phase-only modulations ${ }^{9,10}$. In the case of phase-only modulation we also need short wavelengths to achieve a $360^{\circ}$ phase range. The setup for elliptically polarized light generation and detection is illustrated in Figure 4. The wave plates WP1 and WP2 introduce retardances of $\Gamma_{l}$ and $\Gamma_{2}$ respectively. The angles of the elements in front of the LCD are referred to the orientation of the director at the input surface (which we suppose that coincides with the $X$-coordinate axis) while the angles of the elements behind the LCD are measured with respect to the orientation of the director at the output surface.

In HDSS typically the data page is introduced as a binary absorption mask onto the SLM, thus binary amplitude modulation is required as given in Fig. 3(e). The fidelity in the reconstruction process increases with the increase in the contrast between the high and the low transmission levels. Binary phase modulation ${ }^{16,17}$ with a phase difference of $\pi$ radians and hybrid ternary modulation $(\mathrm{HTM})^{14,15}$ have also been proposed to represent the data page. These two schemes produce Fourier transforms with a low or non-existing DC peak ${ }^{14-17}$. This allows for an optimum use of the dynamic range of the holographic recording material and provides a higher discrimination capability when applying the holographic memory for associative retrieval of information.

In what follows we show how applying the model in Ref. 11, which makes use of the characterization results provided in Section 2, and applying a computer search we are able to find the different modulation regimes useful in the various applications. We use quarter wave plates in the setup. The measured retardances for the two wave plates are $\Gamma_{I}=88$ degrees and $\Gamma_{2}=90$ degrees respectively for the wavelength $532 \mathrm{~nm}$, which is the wavelength that we intend to use in the holographic setup. In the optimization process the magnitudes, $\eta_{1}, \eta_{2}, \varphi_{1}$ and $\varphi_{2}$, are left as free parameters. We assign initial arbitrary values to the angles $\eta_{1}, \eta_{2}, \varphi_{1}$ and $\varphi_{2}$, and we calculate the transmittance $T$ and the phase-shift $\psi$ as a function of the gray level. The optimization criterion to be applied depends on the modulation regime we are interested on.

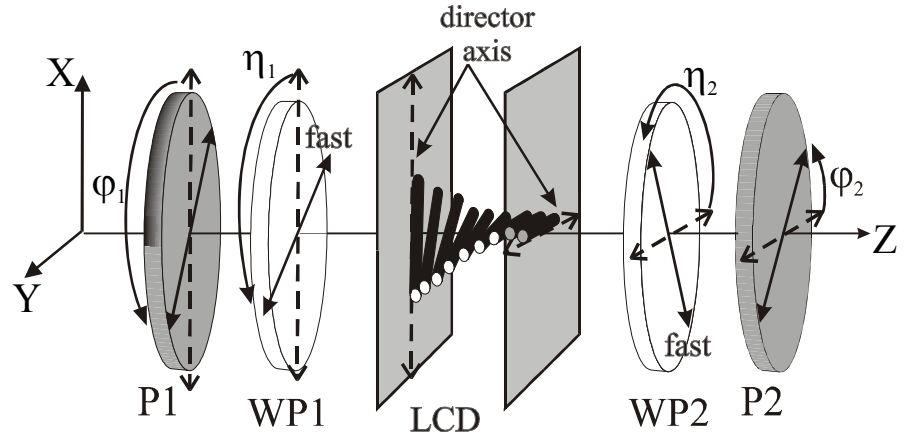

Figure 4. Scheme for the generalized elliptical light set-up.

In the optimum amplitude-only configuration we are interested in maximizing the contrast between the maximum and minimum transmission while minimizing the phase-shift modulation depth. In what follows we define the contrast as the ratio,

$$
\text { Contrast }=(\text { High Intensity Transmission }) /(\text { Low Intensity Transmission })
$$

Table 2.Orientation of the external polarization elements for the optimum modulation configurations calculated in this work.

\begin{tabular}{|c|c|c|c|c|}
\hline Modulation Regime & $\left.\boldsymbol{\varphi}_{\mathbf{1}} \mathbf{(}^{\mathbf{}}\right)$ & $\left.\boldsymbol{\eta}_{\mathbf{1}} \mathbf{(}^{\mathbf{}}\right)$ & $\boldsymbol{\eta}_{\mathbf{2}}\left({ }^{\boldsymbol{}}\right)$ & $\boldsymbol{\varphi}_{\mathbf{2}}\left({ }^{\boldsymbol{o}}\right)$ \\
\hline Amplitude-only & 46.7 & --- & --- & 49.5 \\
\hline Phase-only & -4.4 & -36.1 & 16.6 & 47.8 \\
\hline Binary-amplitude & \multicolumn{4}{|c|}{ Amplitude-only configuration: gray=10 and 210} \\
\hline Binary-phase & -37.4 & 0.0 & 0.0 & 37.6 \\
\hline HTM-1 (532 nm) & -18.2 & 16.3 & -73.1 & -100.4 \\
\hline HTM-2 (532 nm) & 152.8 & 126.1 & 127.2 & -0.1 \\
\hline HTM-3 (458 nm) & 117.3 & 120.7 & 135.6 & 6.3 \\
\hline
\end{tabular}


In Fig. 5(a) and 5(b) we show respectively the normalized intensity and the phase-shift curves as a function of the gray level for the optimum amplitude-only modulation that can be achieved with our LCD. These results have been obtained considering only linear polarizers. The circles are the experimental values and the continuous line is the simulation provided by the model. We observe the excellent agreement between experiment and theory. We use the double-slit interferometer ${ }^{24}$ approach to measure the phase-shift. We have measured the phase-shift till gray level 60 since for lower gray levels the visibility of the fringes was very low. We can say that this is an amplitude-only regime. If we consider the addition of wave plates the phase modulation range can be further decreased ${ }^{9}$.

The lowest and the highest transmission values in Fig. 5 occur respectively at gray levels 10 and 210. The contrast between these two levels is very high, exceeding 1:6000. In Ref. 15 they calculate that an acceptable raw BER can be obtained with a contrast of 1:20, even though the system performance can be further improved for higher contrast values. Therefore, we have obtained a good binary-amplitude modulation regime to display binary absorption data pages in a HDSS. The angles for the linear polarizers in this configuration are summarized in Table 2.
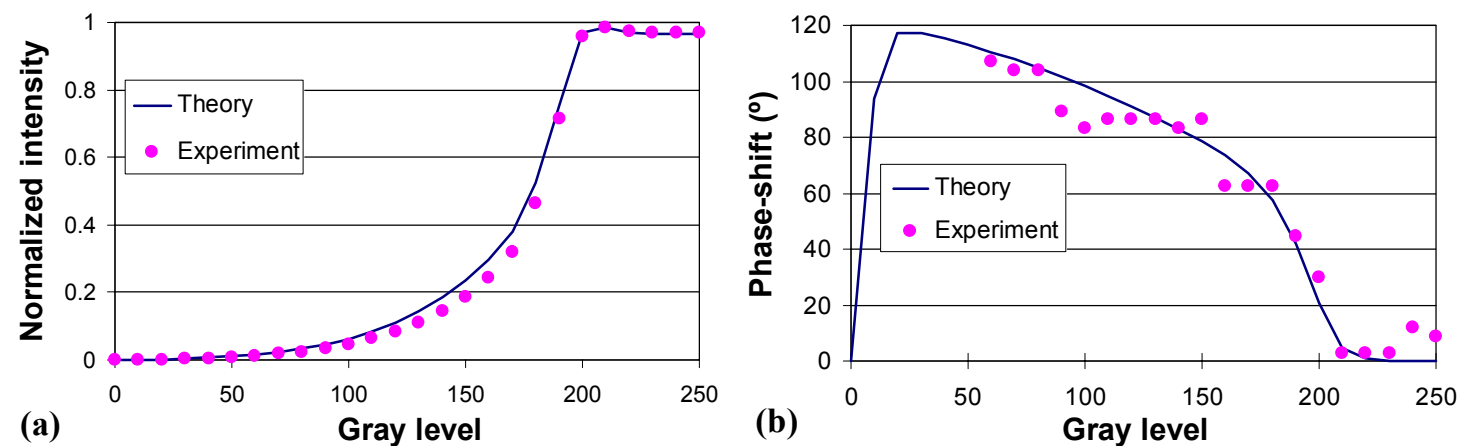

Figure 5. Amplitude-only modulation. (a) Normalized intensity. (b) Phase-shift. The symbols correspond to experimental measurements and the lines correspond to predicted values. We can use gray level 10 as the low transmission level and gray level 210 as the high transmission level for binary-amplitude modulation with a contrast exceeding 1:6000.

In Fig. 6(a) and 6(b) we show respectively the normalized intensity and the phase-shift curves as a function of the gray level for the optimum phase-only modulation that can be achieved with our LCD. The angles for the linear polarizers and quarter wave plates are given in Table 2. As in Fig. 5 we observe the excellent agreement between experiment and theory. We can also see that we are able to obtain a phase-only modulation nearly ideal, with a highly constant intensity response and a phase-shift covering $360^{\circ}$. The intensity transmission has a value about 0.1 . This low value can be a problem in applications where energetic efficiency is a priority. The constraints to obtain phase-only modulation loosen if the LCD is thicker and/or we use shorter wavelengths ${ }^{9}$. In any of these two cases we would be able to obtain optimum phase-only configurations showing a higher intensity transmission.
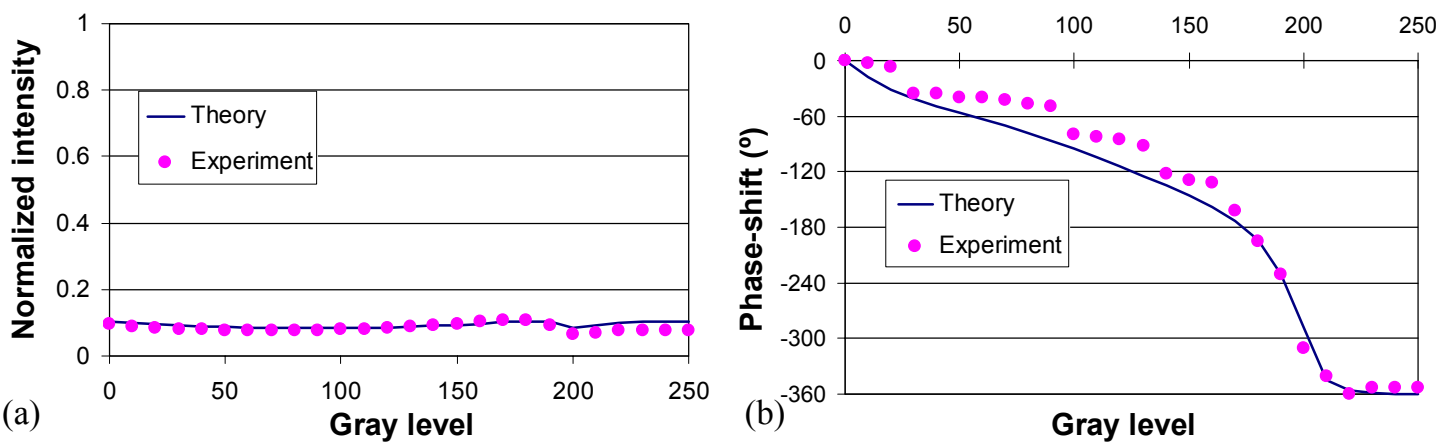

Figure 6. Phase-only modulation. (a) Normalized intensity. (b) Phase-shift. The symbols correspond to experimental measurements and the lines correspond to predicted values. 
The excellent agreement between experimental and theoretical values in Fig. 5 and 6 demonstrates that the model we use together with the values obtained in the characterization in Section 2 allow for an accurate theoretical prediction of the complex amplitude modulation of the LCD for arbitrary orientations of the external polarization elements. In what follows we use this predictive capability of the model to calculate through a computer search some other modulation configurations without the need to compare with experimental values.

First, we look for a binary-phase modulation regime with a high efficiency and able to provide two phase levels with a difference of $180^{\circ}$. In Fig. 7 (a) and $\left.7(b)\right)$ we show the theoretically predicted normalized intensity and phase-shift. We observe (Fig. 7(a)) that the transmission is almost flat and maximum. The phase-shift modulation depth is close to $270^{\circ}$. We can use gray level 0 and gray level 190 as the two levels for binary-phase modulation with a phase difference of $180^{\circ}$. The intensity uniformity between these two levels is $0.5 \%$. We define intensity uniformity as,

$$
\text { Uniformity }=100 \mathrm{x} \text { (Intensity Level1 - Intensity Level2)/(Average Intensity of the two levels) }
$$
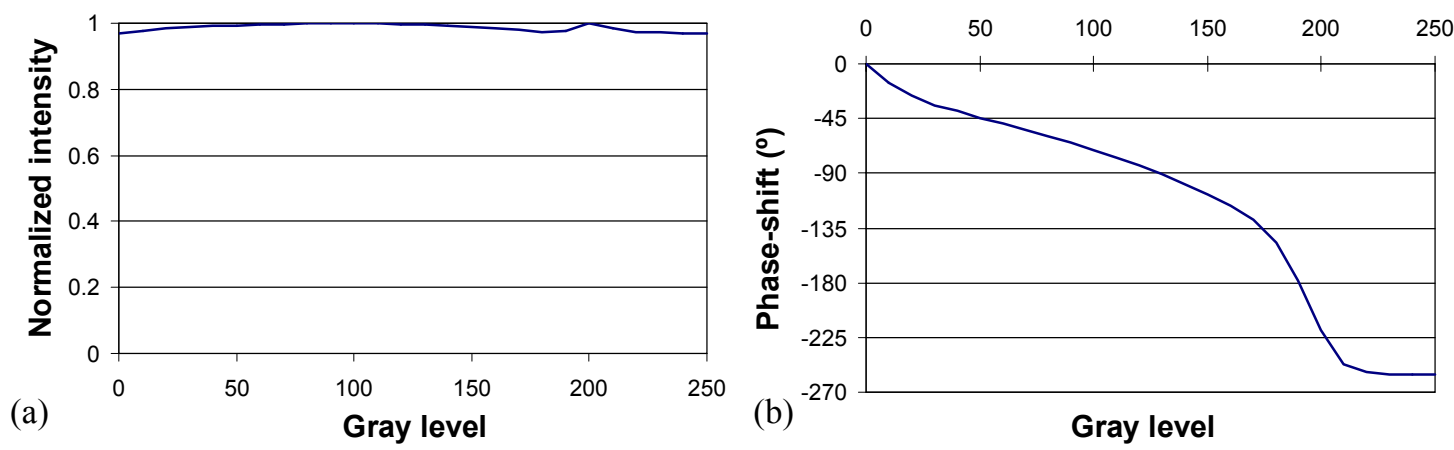

Figure 7. Phase-only modulation with a high transmission efficiency. (a) Normalized intensity. (b) Phase-shift. This is a theoretically predicted result. We can use gray level 0 and gray level 190 as the two levels for binary-phase modulation with a phase difference of $180^{\circ}$ and an intensity uniformity of $0.5 \%$.

Next we focus in the optimization to obtain HTM. For this modulation regime the LCD is required to show two gray levels with a similar transmission and with a $180^{\circ}$ phase difference (representing the white level in the data page) and a low transmission level (representing the black level). The contrast between the white and the black level should be at least 1:20 to produce an acceptable raw BER ${ }^{15}$. In Fig. 8(a) and 8(b)) we show the theoretically predicted normalized intensity and phase-shift. It corresponds to the configuration HTM-1 in Table 2. The figure of merit established for the computer search looked for a low transmission value as low as possible in the mid gray level region with the two high transmission levels at each side of this minimum, imposing a phase difference as close as possible to $180^{\circ}$ and a similar transmission value. This is the criteria followed in Ref. 14. This is accomplished by gray level 130 and 210 as the white levels, and gray level 190 as the black level. Intensity uniformity between the white levels is lower than $10^{-5} \%$, their phase difference is $180^{\circ}$, and the contrast between the white levels and the black level is 1:62. In principle these are good values, especially if we compare with the results in Ref. 14 where the phase difference was merely $132^{\circ}$. The transmission value for the white levels is lower than 0.1. As with Fig. 6 this is a low value when energetic efficiency of the system is a priority, as it is the case in commercial HDSS. However it can still be a useful configuration in research labs to prove system architectures or to investigate new holographic recording materials.

Now we change the figure of merit to obtain HTM. We consider, as in Fig. 9 in Ref. 15, a low transmission value as low as possible at an extreme of the gray level scale. In Fig. 9(a) and 9(b) we show the theoretically predicted normalized intensity and phase-shift. It corresponds to the configuration HTM-2 in Table 2. The black level is at gray level 250 and the two white levels are at gray levels 0 and 190. Intensity uniformity between the white levels is lower than $0.003 \%$, their phase difference is $180^{\circ}$, and the contrast between the white levels and the black level is extremely high with a value of $1: 4.5 \cdot 10^{6}$. The results are largely better than in configuration HTM-1 (Fig. 8) and also better than in Ref. 15 where they report a contrast of 1:200, and a phase-difference of $180^{\circ}$. The transmission value for the white levels is now 0.18 . This is double than in HTM-1 but it is still not as good as in Ref. 15, where they report a transmission value of 0.8 . 

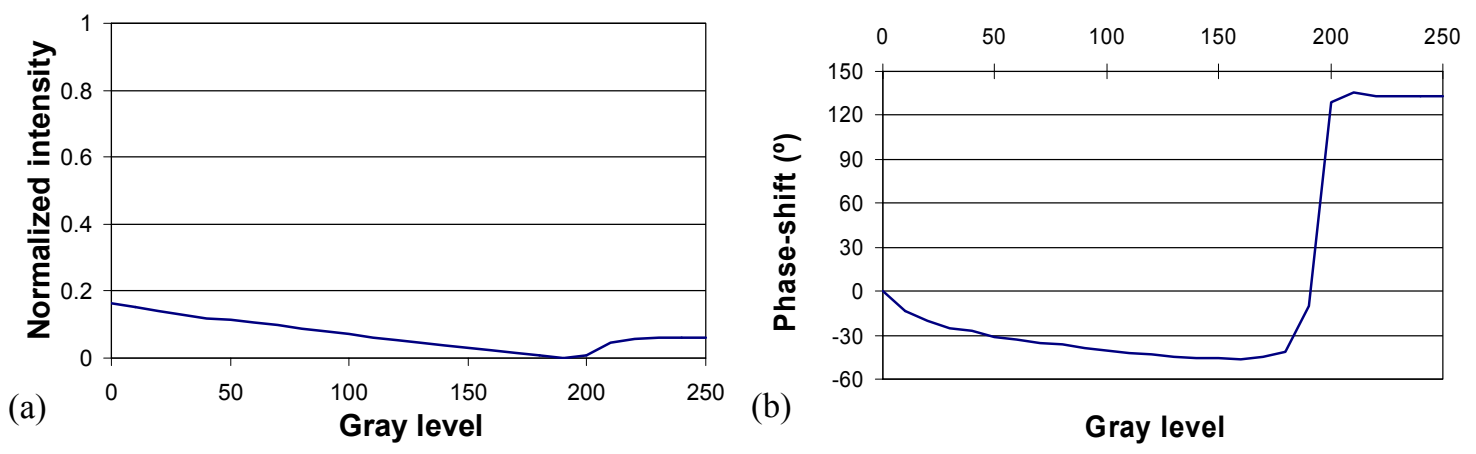

Figure 8. HTM modulation (configuration HTM-1). (a) Normalized intensity. (b) Phase-shift. This is a theoretically predicted result. We can use gray level 130 and gray level 210 as the white levels and gray level 190 as the black level. Intensity uniformity and phase difference between the white levels is lower than $10^{-5} \%$ and $180^{\circ}$ respectively, whereas the contrast between the white levels and the black level is 1:62.
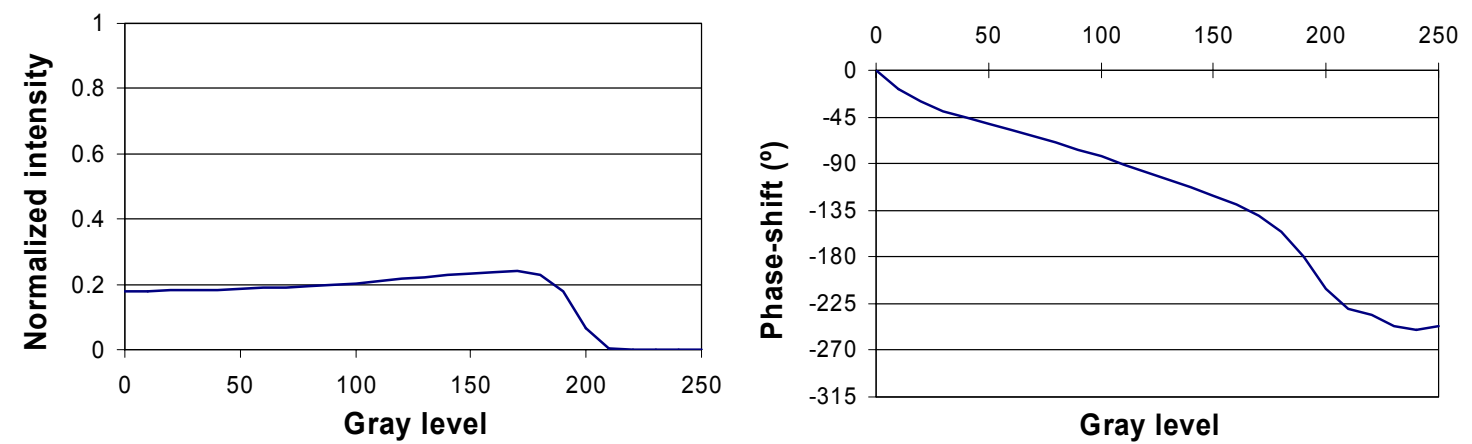

Figure 9. HTM modulation (configuration HTM-2). (a) Normalized intensity. (b) Phase-shift. This is a theoretically predicted result. We can use gray level 0 and gray level 190 as the white levels and gray level 250 as the black level. Intensity uniformity and phase difference between the white levels is lower than $0.003 \%$ and $180^{\circ}$ respectively, whereas the contrast between the white levels and the black level is $1: 4.5 \cdot 10^{6}$.

There is a sensible difference between the experiments in the present work, where we consider a wavelength of $532 \mathrm{~nm}$, and the experiments in Ref. 15, where they use a wavelength of $406 \mathrm{~nm}$. A shorter wavelength allows for a larger birefringence modulation depth, which loosens the constraints to obtain certain modulation regimes, in special the phaseonly regime ${ }^{9,10}$. Next, we want to check if a larger birefringence modulation depth would allow for a more optimal HTM configuration. To this goal we consider an illumination wavelength of $458 \mathrm{~nm}$. We know from previous works ${ }^{9,11}$ that for the LCD model LCX012BL $\beta$ and $\delta$ birefringences scale by a factor of 1.24 for $458 \mathrm{~nm}$ in comparison with the birefringences for $532 \mathrm{~nm}$. We consider the scaled birefringence values to calculate the transmittance $T$ and the phaseshift $\psi$ as a function of the gray level for $458 \mathrm{~nm}$. The figure of merit for the computer search considers a low transmission value as low as possible at an extreme of the gray level scale with the two high transmission levels at the other extreme, imposing a phase difference as close as possible to $180^{\circ}$ and a similar transmission value. This is the figure of merit used in HTM-2, that has proven more satisfactory than the figure of merit used in HTM-1. In Fig. 10(a) and 10(b)) we show the theoretically predicted normalized intensity and phase-shift. It corresponds to the configuration HTM-3 in Table 2. The black level is at gray level 240 and the two white levels are at gray levels 0 and 180 . Intensity uniformity between the white levels is lower than $0.0004 \%$, their phase difference is $180^{\circ}$, and the contrast between the white levels and the black level is $1: 10^{9}$. The transmission value for the white levels is 0.6 . We have obtained better values for the various parameters in comparison with HTM-2, especially we have greatly increased the transmission value for the white levels. Clearly a larger birefringence modulation depth at $458 \mathrm{~nm}$ allows for a more flexible trade-off between the various parameters of interest.

At this point we note that the contrast values between the white and the black levels reported in relation with Fig. 5, 8, 9 and 10 are calculated using the theoretical intensity. Experimentally we have verified that contrast values exceeding 1:1000 are feasible with the LCD and the polarization elements used in this work, which are actually excellent values. 

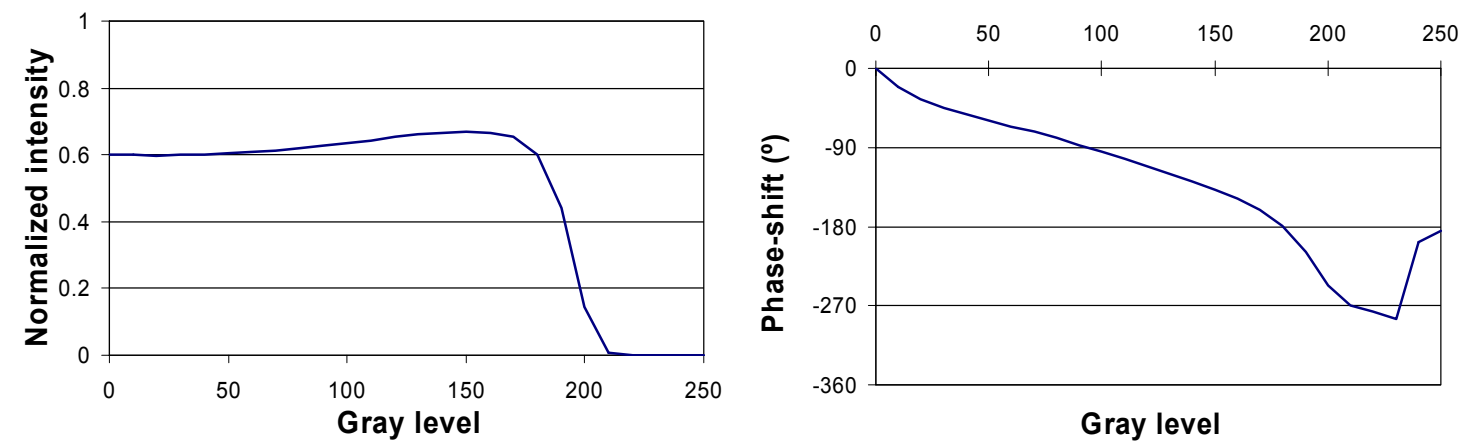

Figure 10. HTM modulation (configuration HTM-3). (a) Normalized intensity. (b) Phase-shift. This is a theoretically predicted result. We can use gray level 0 and gray level 180 as the white levels and gray level 240 as the black level. Intensity uniformity and phase difference between the white levels is lower than $0.0004 \%$ and $180^{\circ}$ respectively, whereas the contrast between the white levels and the black level is $1: 10^{9}$.

In Fig. 11 we plot a complex plane representation of the HTM configurations presented in Fig. 8, 9 and 10. In Fig. 11(a), (b) and (c) are represented the configurations HTM-1, HTM-2 and HTM-3 respectively. The complex plane representation allows for a geometrical interpretation of the figures of merit used to obtain the different configurations. In the configuration HTM-1 we imposed a low transmission value in the mid gray level range as in Ref. 14. In the configurations HTM-2 and HTM-3 we imposed the low transmission value at an extreme of the gray level scale as in Ref. 15. We note that the two criteria produce two different sorts of geometrical trajectories. HTM-1 is almost a straight line passing from the 4 rth to the $2^{\text {nd }}$ quadrant through the origin. HTM-2 and HTM-3 are highly coupled amplitude and phase curves, with a spiral shape. From the results obtained we demonstrate that the criterion for HTM-2 and HTM-3 is superior: actually, coupled amplitude and phase modulation is a natural regime exhibited by LCDs as commented in Fig. 3. The constraints of the criterion proposed by Jang and Shin ${ }^{14}$ are further discussed in Ref. 15.

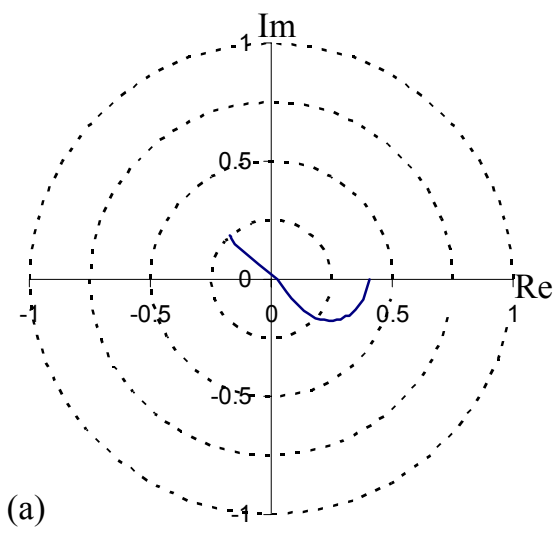

(b)

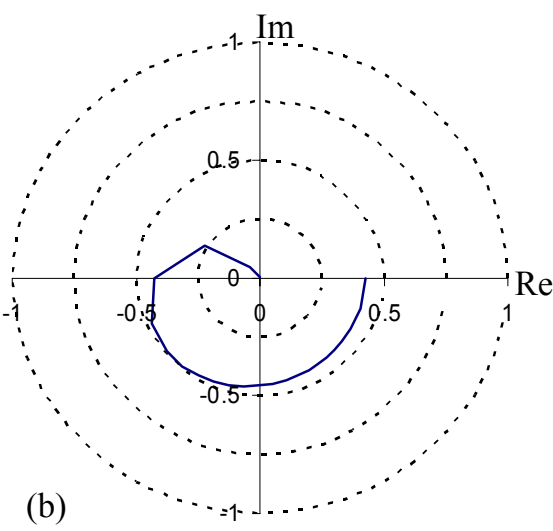

(c)

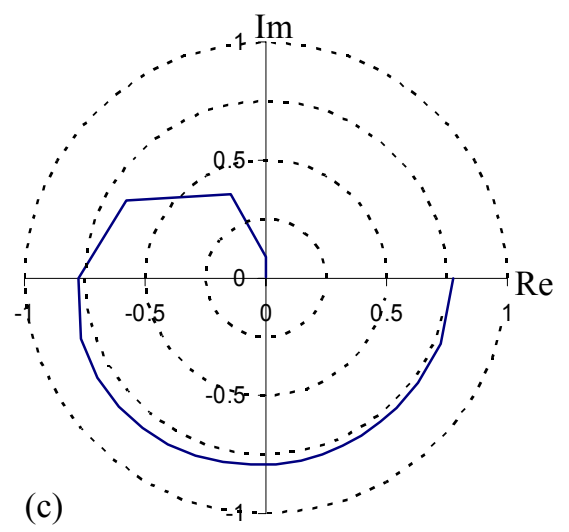

Figure 11. Complex plane representation of the HTM configurations previously obtained. (a) HTM-1. (b) HTM-2. (c) HTM-3.

\section{HOMOGENIZATION OF THE FOURIER TRANSFORM}

In this Section we want to compare the degree of homogenization of the Fourier transform (FT) provided by each of the modulation schemes analyzed in Section 3. To this goal we consider binary random data pages with a size of $256 \times 256$ pixels and with an equal number of 0 's and 1's, which simulate an actual data page addressed in a holographic memory setup. Using the MATLAB software we produce different realizations of the binary random data pages and we calculate their FT. A zoom of one of the random realizations of the page is shown in Fig. 12(a). In Fig. 12(b) we represent the three classes of pixels in a binary data page implementing the HTM scheme. 


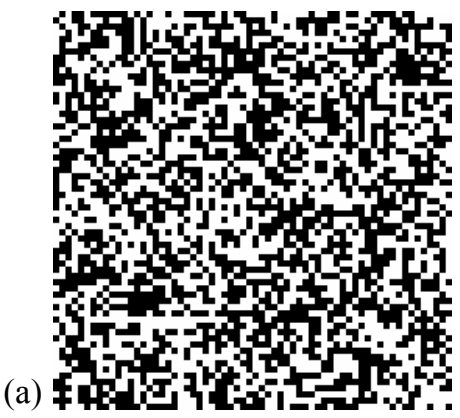

(b)

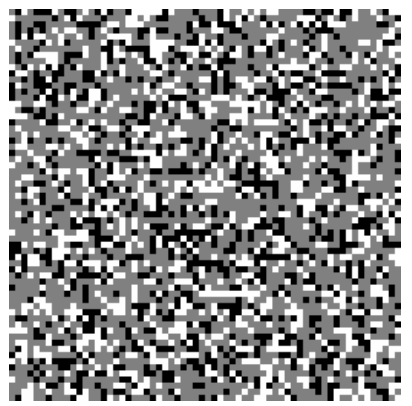

Figure 12. Zoom of one of the realizations of the randomized binary data page with an equal number of 0's and 1's. (a) Black and white represent the 0's and 1's. (b) In the case of the HTM we need 3 gray levels to represent graphically the 0's (gray pixels) and the 1's (black and white pixels represent the phase difference of $180^{\circ}$ ).

To analyse the FT plane we have considered a series of parameters. We have controlled the peak intensity value (peak) and its position, and the transmission efficiency (efficiency), defined as the ratio between the energy at the FT plane to the total incident energy. We have also calculated the normalized degree of inhomogenity, defined as the ratio between the peak intensity to the average intensity in the FT plane normalized by the value of this ratio for the binary phase regime. The closest to one the value of this parameter the most homogenously is the energy distributed in the Fourier plane. In Table 3 we summarize the values for these parameters and for the various modulation regimes.

Table 3.Values of the parameters to analyse the results at the FT plane for the different modulation regimes.

\begin{tabular}{|c|c|c|c|}
\hline Modulation Regime & $\begin{array}{c}\text { Peak in arbitrary units } \\
\text { (Position in pixel units) }\end{array}$ & Efficiency & $\begin{array}{c}\text { Normalized } \\
\text { inhomogenity }\end{array}$ \\
\hline Binary amplitude (0,1) & $1.1 \times 10^{9}(129,129)$ & 0.5 & $3.3 \times 10^{3}$ \\
\hline Binary phase (0,pi) & $5.7 \times 10^{5}(205,121)$ & 1 & 1 \\
\hline HTM ideal & $3.0 \times 10^{5}(9,118)$ & 0.5 & 1 \\
\hline HTM-1 (532 nm) & $8.3 \times 10^{5}(129,129)$ & 0.023 & 56 \\
\hline HTM-2 (532 nm) & $5.7 \times 10^{4}(251,26)$ & 0.09 & 1 \\
\hline HTM-3 (458 nm) & $2.0 \times 10^{4}(190,215)$ & 0.3 & 1 \\
\hline
\end{tabular}

In the first three rows we show the results respectively for the ideal binary amplitude, ideal binary phase and ideal HTM regimes. Binary amplitude is the regime typically used in most of the holographic memory setups. We see that the peak intensity (second column) is various orders of magnitude higher in comparison with the rest of the modulation schemes. This peak intensity is located at the centre of the Fourier plane, position $(129,129)$, thus it is produced by the DC term. The peak intensities for the ideal binary phase and the ideal HTM do not occur at the zero frequency. Actually, at the zero frequency the intensity value tends to be various orders of magnitude lower than the peak intensity. In these two regimes the position for the peak intensity changes with each of the realizations of the random binary data page, even though the peak intensity maintains a similar value. Since all the pixels are transparent the efficiency (column 3) for the ideal binary phase regime is double than it is for the binary amplitude and ideal HTM regimes. As it is expected we see that the distribution of the energy in the Fourier plane (column 4) is not homogenous at all in the case of the binary amplitude regime.

In the last three rows we compare the results provided when we consider real configurations for the HTM. We use the values for the transmittances and phase-shifts given in Section 3 for the configurations HTM-1, HTM-2 and HTM3. We observe that the peak intensity for the configuration HTM-1 corresponds to the DC term as in the case of the binary amplitude regime, even though the FT plane is two orders of magnitude more homogenous (column 4). In the case of the configurations HTM-2 and HTM-3 the FT plane is as homogenous as the ideal. These two configurations presented excellent contrast ratios in Section 3. With respect to the efficiency, the configurations HTM-2 and HTM-3 have values of 0.09 and 0.3 which are respectively $80 \%$ and $40 \%$ lower than the efficiency for the ideal HTM case. From the 
discussion in Section 3, thicker LCDs with a larger birefringence modulation depth would allow to obtain more efficient configurations.

In Fig. 13(a) and (b) we show the two different sorts of Fourier planes obtained with the various modulation configurations in Table 3. We represent the modulus of the FT. In Fig. 13(a) we show the FT plane when using the binary amplitude and the HTM-1 schemes. In both cases a high DC peak in the centre saturates the dynamic range of the image, thus the rest of the plane appears totally black. Fig. 13(b) can be obtained with binary phase, ideal HTM, HTM-2 and HTM-3. In all these cases the energy is homogenously distributed and no DC peak can be appreciated.

(a)

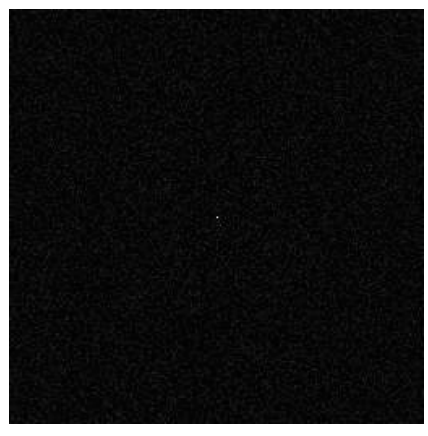

(b)

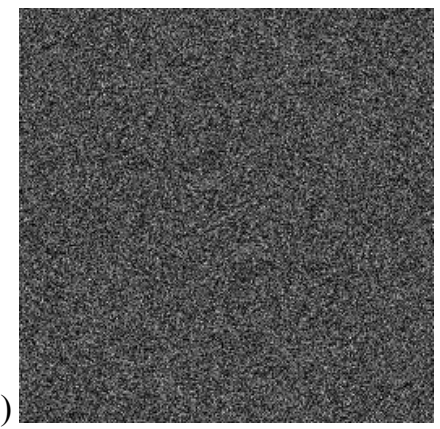

Figure 13. Modulus of the FT of the random binary data page. (a) FT plane obtained in the case of the binary amplitude and HTM-1 configurations. (b) FT plane obtained in the case of the binary phase, ideal HTM, HTM-2 and HTM-3 configurations.

As a final remark, we want to note that the analysis in this work is focused to increase the performance of the holographic memory setup in our lab. One of the main components in this setup is the holographic recording material. We use photopolymer compounds based on a polyvinil alcohol (PVA) matrix, and acrylamide (AA) monomers ${ }^{25-27}$. They have been extensively studied, as they combine good optical properties, ease of fabrication, self-development capability and they offer the capability to establish thick recording layers.

\section{CONCLUSIONS}

We have presented the characterization and the optimization of the phase and amplitude modulation of a commercial LCD applied to HDSS. We have provided the results for the characterization of the parameters of LCD according to an approximate model describing the modulation of the anisotropic properties of the LCD as a function of the applied voltage. This model has proven in the literature to provide accurate calculations of the amplitude and phase-shift modulations of the LCD. We have used the model to obtain the optimum configurations available with the LCD for the modulation regimes typically demanded in the literature in the application to HDSS: binary amplitude, binary phase and HTM. In the case of HTM we obtained that highly coupled amplitude and phase modulation regimes are necessary. We have also demonstrated that a larger birefringence modulation depth allows to obtain more optimal HTM configurations, with a higher transmittance. We have also analyzed the performance of these configurations to perform the Fourier transform of a binary data page. We see that the optimum configurations obtained for the binary phase and the HTM regimes provide a homogenous distribution of the energy in the Fourier plane.

\section{ACKNOWLEDGMENTS}

This work was supported by the Spanish Ministerio de Educación y Ciencia (grants FIS2005-05881-C02-01 and FIS2005-05881-C02-02), and from Generalitat Valenciana (grant GV06-007).

\section{REFERENCES}

1. P. Yeh and C. Gu, Optics of liquid crystal displays, John Wiley \& Sons, (1999).

2. V. Laude, "Twisted-nematic liquid-crystal pixelated active lens", Opt. Commun. 153, 134-152 (1998). 
3. J. A. Davis, J. C. Escalera, J. Campos, A. Márquez, M. J. Yzuel and C. Iemmi, "Programmable axial apodizing and hyperresolving amplitude filters using a liquid crystal spatial light modulator”, Opt. Lett. 24, 628-630 (1999).

4. A. Márquez, C. Iemmi, J. C. Escalera, J. Campos, S. Ledesma, J. A. Davis and M. J. Yzuel, "Amplitude apodizers encoded onto Fresnel lenses implemented on a phase-only spatial light modulator", Appl. Opt. 40, 2316-2322 (2001).

5. A.Márquez, C. Iemmi, J. Campos and M. J. Yzuel, “Achromatic diffractive lens written onto a liquid crystal display", Opt. Lett. 31, 392-394 (2006).

6. H.J. Coufal, D. Psaltis and B.T. Sincerbox, eds., Holographic Data Storage, Springer-Verlag, Berlin, (2000).

7. M. L. Álvarez, A. Márquez, L. A. Puerta, R. Estévez, E. Fernández, A. Beléndez and I. Pascual, "Caracterización de una pantalla de cristal líquido de baja resolución para su uso como modulador óptico", Opt. Pura Apl. 40, 95-103 (2007).

8. J. L. de Bougrenet de la Tocnaye and L. Dupont, "Complex amplitude modulation by use of liquid-crystal spatial light modulators,” Appl. Opt. 36, 1730-1741 (1997).

9. A. Márquez, C. Iemmi, I. Moreno, J. A. Davis, J. Campos and M. J. Yzuel, "Quantitative prediction of the modulation behavior of twisted nematic liquid crystal displays based on a simple physical model," Opt. Eng. 40, 2558-2564 (2001).

10. J. Nicolás, J. Campos and M. J. Yzuel, "Phase and amplitude modulation of elliptic polarization states by nonabsorbing anisotropic elements: application to liquid crystal devices," J. Opt. Soc. Am. A 19, 1013-1020 (2002).

11. A. Márquez, J. Campos, M. J. Yzuel, I. Moreno, J. A. Davis, C. Iemmi, A. Moreno and A. Robert, "Characterization of edge effects in twisted nematic liquid crystal displays", Opt. Eng. 39, 3301-3307 (2000).

12. R. K. Kostuk, M. P. Bernal and Q. Cao, "Beam conditioning techniques for holographic recording systems", in Holographic Data Storage, H. J. Coufal, D. Psaltis, and G. T. Sincerbox, eds., Springer-Verlag, Berlin, (2000).

13. M. P. Bernal, G. W. Burr, H. Coufal, J. A. Hoffnagle, C. M. Jefferson, R. M. Macfarlane, R. M. Shelby and M. Quintanilla, "Experimental study of the effects of a six-level phase mask on a digital holographic storage system", Appl. Opt. 37, 2094-2101 (1998).

14. J.-S. Jang and D.-H. Shin, "Optical representation of binary data based on both intensity and phase modulation with a twisted-nematic liquid-crystal display for holographic digital data storage", Opt. Lett. 26, 1797-1799 (2001).

15. J. Reményi, P. Várhegyi, L. Domján, P. Koppa and E. Lorincz, "Amplitude, phase, and hybrid ternary modulation modes of a twisted-nematic liquid-crystal display at_400 nm", Appl. Opt. 42, 3428-3434 (2003).

16. R. John, J. Joseph and K. Singh, "Holographic digital data storage using phase-modulated pixels", Optics and Lasers in Engineering 43, 183-194 (2005).

17. J. Joseph and D. A. Waldman, "Homogenized Fourier transform holographic data storage using phase spatial light modulators and methods for recovery of data from the phase image", Appl. Opt. 45, 6374-6380 (2006).

18. C. Soutar and K. Lu, "Determination of the physical properties of an arbitrary twisted-nematic liquid crystal cell", Opt. Eng. 33, 2704-2712 (1994).

19. J. A. Davis, D. B. Allison, K. G. D'Nelly, M. L. Wilson and I. Moreno, "Ambiguities in measuring the physical parameters for twisted-nematic liquid crystal spatial light modulators," Opt. Eng. 38, 705-709 (1999).

20. J. A. Davis, P. Tsai, K. G. D'Nelly and I. Moreno, "Simple technique for determining the extraordinary axis direction for twisted-nematic liquid crystal spatial light modulators", Opt. Eng. 38, 929-932 (1999).

21. A. Márquez, Accurate predictive model for twisted nematic liquid crystal devices. Application for generating programmable apodizers and Fresnel lenses. Ph.D. Thesis, Universitat Autònoma de Barcelona, pp. 35-36, (2001).

22. D. W. Berreman, "Optics in smoothly varying anisotropic planar structures: Application to liquid-crystal twist cells", J. Opt. Soc. Am. 63, 1374-1380 (1973).

23. D. W. Berreman, "Dynamics of liquid-crystal twist cells," Appl. Phys. Lett. 25, 12-15 (1974).

24. A. Bergeron, J. Gauvin, F. Gagnon, D. Gingras, H. H. Arsenault and M. Doucet, "Phase calibration and applications of a liquid-crystal spatial light modulator", Appl. Opt. 34, 5133-5139 (1995).

25. M. Ortuño, S. Gallego, C. García, C. Neipp, A. Beléndez and I. Pascual, "Optimization of a $1 \mathrm{~mm}$ thick PVA/acrylamide recording material to obtain holographic memories: method of preparation and holographic properties", Applied Physics B 76, 851-857 (2003).

26. C. Neipp, S. Gallego, M. Ortuño, A. Márquez, M.L. Álvarez, A. Beléndez and I. Pascual, "First-harmonic diffusionbased model applied to a polyvinyl-alcohol-acrylamide-based photopolymer", JOSA B 20, 2052-2060 (2003).

27. S. Gallego, M. Ortuño, C. Neipp, A. Beléndez and I. Pascual, "Influence of fringe visibility on the characteristics of holograms recorded in photopolymer material", Optik 114, 401-406 (2003). 